- Bilateral pedal edema

- Loss of appetite

If any one parameter was present the child was started with F-75 diet every 2 hours for 48 hours followed by F-100 diet every 4 hours for the next 48 hours. If none of the parameters were present children were started directly with F100 diet, once the discharge criteria were met, discharged and followed up every fortnightly for 8 weeks.

Discharge Criteria:

- $15 \%$ Weight gain from admission weight or weight on the day free of oedema

- Absence of Bilateral Oedema for at least 10 days

Results 68 subjects were enrolled, most common age group 24-35 months, 55.9\%-boys 44.1\%-girls, 91.2\% were term $8.8 \%$ were pre term babies.

$36.8 \%$ - exclusively breastfed for 6 months. 33.8\%breastfed for less than 6 months (Avg-5.1 months) 29.4\% breastfed for more than 6 months (Avg-7.8 months).

There was a statistically significant increase in the mean weight as the mean weight at admission was $8.29 \pm 1.49$ and at discharge was $8.72 \pm 1.51$ ( $\mathrm{p}$ value $<0.001$ ).

The height at the time of admission and discharge was $81.24 \pm 8.11$ and $81.34 \pm 8.13$ respectively. There was no significant difference in mean height of patients ( $p$ value$0.676)$.

On 4th follow up 52 (76.4\%) children had attained normal weight for height with a $Z$ score of $<-2$ SD.

13 children had shifted from SAM to MAM status, but three children persisted as SAM.

Conclusion NRCs had a definite positive impact on the Weight for Height Z Score of children after 4 follow ups. Reinforcement of NRC diet and close follow up is required in children who suffer from acute illnesses, to prevent reverting back to SAM.

\section{G274 DELTA 12S INDEX AS A SCREENING TOOL FOR OBSTRUCTIVE SLEEP APNOEA IN CHILDREN}

L See, AKS McBride, M Shah, S Wilkinson, M DeKrujif, O Narayan. Paediatric Respiratory Department, Royal Manchester Children's Hospital, Manchester, UK

\subsection{6/archdischild-2020-rcpch.237}

Introduction and Aims Obstructive sleep apnoea (OSA) affects up to $5.7 \%$ of children and is associated with significant morbidity. Clinical assessment is subjective and an unreliable predictor of OSA. ${ }^{1}$ To date, no single parameter has been validated for predicting OSA in otherwise healthy children. The gold standard investigation in suspected OSA is polysomnography (PSG), which is labour- and resource-intensive, requires expert interpretation and can be poorly tolerated. Nocturnal oximetry studies are cost effective, widely available and well tolerated.

Delta 12s, a measure of oxygen saturation variability in oximetry studies, has been validated for the prediction of OSA in adults ${ }^{2}$ and in children with Down's syndrome ${ }^{3}$ but has not been studied in children without co-morbidities. We aimed to demonstrate whether the delta $12 \mathrm{~s}$ index predicts OSA in children without Down's syndrome.

Methods We identified 500 sequential patients who underwent adeno-tonsillectomy at our centre from electronic records. Exclusion criteria were children with Down's syndrome and those with no recorded oximetry study. It was then determined which of these children had OSA (defined as OSA diagnosed by an ENT surgeon or respiratory paediatrician, or an oxygen desaturation index $\geq 5$ ). Delta $12 \mathrm{~s}$ index derived from these children's oximetry data was retrospectively analysed for its ability to predict OSA.

Results $155 / 500$ children met criteria for inclusion. In this sample, 78 were determined to have OSA and 19 were not. The remaining 58 were undetermined. The relationship between Delta $12 \mathrm{~s}$ and OSA is highly significant $(\mathrm{p}<0.001)$. A delta $12 \mathrm{~s}$ index of $\geq 0.46$ was shown to predict OSA with a sensitivity of $97 \%$ and specificity $79 \%$.

Conclusion Delta $12 \mathrm{~s}$ is a simple parameter that can be used by general paediatricians as a screening tool to identify symptomatic children without Down's syndrome with a high likelihood of OSA.

\section{REFERENCES}

1. Dehlink E, et al. J Thorac Dis. 2016; 8: 224-235.

2. Magalang UJ, et al. Chest2003;124:1694-707

3. Hill CM, et al. Arch Dis Child 2018;103:962-7

\section{G275(P) HOME OXYGEN THERAPY: A DRUG LIKE ANY OTHER}

M Kamal, L Farrell, R O'Reilly. Pediatrics Respiratory Medicine, CHI Crumlin (OLCHC), Dublin, Ireland

\subsection{6/archdischild-2020-rcpch.238}

Home oxygen services play a vital role in supporting children with breathing difficulties with a wide variety of underlying medical conditions, many of whom will wean out of oxygen over time. However, unlike other medical prescriptions which need to be reviewed 6 monthly, oxygen continues to be supplied until the prescriber requests in writing for it to be removed. Our aim was to review oxygen prescribed in OLCHC.

Methods Air Liquide, the main supplier of oxygen services, provided a list of all children prescribed oxygen between 1/1/ 2010 and $31 / 1 / 2019$ by OLCHC and who are currently supplied oxygen. Clinical records were reviewed for data.

Results 82 patients were prescribed oxygen over 8 years and are living all over the Republic of Ireland. Almost half (43/ 82) were prescribed oxygen for respiratory causes. The other causes were congenital heart disease $(n=13)$, Trisomy 21 $(n=10)$ and other $(n=16)$. The median age was 3 (range 0.1-20.3) years at time of oxygen prescription. The length of time on oxygen was 2.7 (range $0.1-9$ ) years. The main prescribers were cardiology, neonates and respiratory. 56/82 children were reviewed in OLCHC within the last 12 months.

Conclusion On review of the notes it can be difficult to assess if children are still using oxygen particularly when many are often followed up by several clinicians and/or multiple hospitals. A formal review process should be put in place for children on oxygen so it can be titrated appropriately and removed in a timely manner when no longer needed.

Discussion Any patient newly prescribed home oxygen therapy will automatically require a 6 monthly prescription as per the standard operating procedure. Improve 6 monthly prescribing of oxygen for patients. Slow introduction of 6 monthly prescribing of home oxygen to return/existing patients. 